\title{
Towards Accountability: The Articulation and Formalization of Fairness in Machine Learning. ${ }^{1}$
}

\author{
Laurens Naudts ${ }^{1}$
}

${ }^{1}$ KU Leuven Centre for IT \& IP Law, Leuven, Belgium

\section{Introduction}

Whether they classify, cluster, filter or prioritise information, machine learning techniques enable Big Data sense-making (Colonna 2013; Diakopoulos 2015). Machine learning processes have been deployed to inform and automate recommender systems , hiring practices, credit scoring, predictive policing, etc. (see also Robinson, Yu, and Rleke 2014). The decision-making processes they guide, whether they are fully automated or not, affect both individuals and groups of individuals. Machine learning is thus increasingly used in ways that exert an influence on the distribution of benefits and burdens across citizens within society, including the opportunities they are granted. As a consequence, the reliance on automated decision-making processes warrants due caution concerning their potential impact. The data science community has not been indifferent to the ethical issues, such as questions concerning equality, privacy and data protection that may arise out of their field. ${ }^{2}$ In recent years, various efforts have been made to embed 'fairness' into the design of machine learning techniques (See inter alia Dwork et al. 2011; Joseph et al. 2016; Dwork et al. 2017; Zafar, Valera, Rodriguez, and Gummadi 2017; Hardt, Price, and Srebro 2016). This 'formalization' of fairness sets as a goal to reduce unfair outcomes that might result out of machine learning practices. Before one can formalize fairness, it must first be articulated. Yet, fairness is not easily captured and many conceptions of what is fair exist. For machine learning, theories of distributive justice in particular might serve as a good ground for articulating fairness. Theories of distributive justice debate the preferred distribution of benefits and burdens among members of society, providing general principles as moral guidance (Jasso, Törnblom, and Sabbagh 2016). As machine learning affects the distribution of benefits and burdens for those affected by automatically guided decision-making, principles of distributive justice might have an important function in informing the future development of fair machine learning approaches.

\footnotetext{
${ }^{1}$ The following is a working draft: all comments and feedback are welcome. This paper was submitted for pre-proceedings to, and won the Best Student Paper Award at, the 2018 IFIP Summer School on Privacy and Identity Management (https://www.ifip-summerschool.org/accepted-papers/)

${ }^{2}$ See inter alia the combined efforts of the Fairness, Accountability and Transparancy in Machine Learning community (FAT/ML); https://www.fatml.org/
} 


\subsection{Problem Statement and Paper Outline}

The paper will first consider how theories of distributive justice have informed, and are important for informing future, developments and research within the field of machine learning. Under the first section, the paper will analyze current fair machine learning approaches. It will be considered whether these approaches are sufficiently adequate in dealing with the ethical concerns that are generally associated with machine learning. After this primary assessment, the paper will discuss how distributive justice could contribute towards future fair machine learning efforts. The goal of this paper is not to provide an extensive overview of, nor a detailed insight into, conceptions of fairness that exist within the domains of political philosophy, legal theory or machine learning. ${ }^{3}$ Rather, it aims to indicate whether or not the ways in which data science currently approaches fairness are sufficient in grasping the ethical challenges posed by machine learning techniques, and whether there is a need to further articulate fairness in machine learning. In this regard, the paper will also explore the barriers that exist with regard to the articulation and formalization of fairness. Finally, the paper will try to provide a preliminary analysis on how the articulation and formalization of fairness can (indirectly) increase the accountability of the actors who deploy machine learning systems.

\section{The Formalization of Fairness}

The machine learning community has been quite aware of the potential dangers their developed techniques might have on fundamental interests, rights and values, such as equality, privacy, data protection, rule of law and democracy. ${ }^{4}$ In reaction, data scientists have tried to develop fair machine learning systems. Fair machine learning relies upon the formalization of fairness: a given notion of fairness is abstracted and embedded into the design of a computational process. In general, this entails that the 'reasoning' and 'learning' processes involved, are developed in such a way that the ultimate outcome of these processes corresponds to what, a priori, has been defined as fair. Amongst current fair machine learning techniques, a main distinction can be made depending on whether these processes aim to achieve parity or preference (Gajane and

\footnotetext{
${ }^{3}$ For a brief overview of fairness within machine learning, see inter alia (Binns 2018 and Gajane 2018)

${ }^{4}$ The Fairness, Accountability and Transparency in Machine Learning (FATML) community aims to bring together researchers and practitioners in order to address the 'novel challenges' machine learning poses towards ensuring amongst others non-discrimination, due process, and understandability of decision-making systems. According to the FATML website, the community aims to provide 'researchers' a venue to critically reflect upon these issues with 'computationally rigorous methods'. In addition, the FATML website provides an extensive overview of scholarship within the field of fair machine learning; available via: https://www.fatml.org/
} 
Pechenizkiy 2017). ${ }^{5}$ Following their taxonomy and overview of the state of the art in fair machine learning, both parity and preference techniques will be further discussed. ${ }^{6}$

\subsection{Parity-based Fairness in Machine Learning}

Parity-based systems draw inspiration from equality as a principle of justice. Where parity, read equality, is achieved, either in treatment or impact, the outcome of the learning system is considered fair. Machine learning can ensure parity treatment by avoiding the use of sensitive attributes, such as gender or ethnicity, in machine learning processes. In essence, sensitive attributes are simply removed from the learning system. In other words, the system remains agnostic, i.e. unaware, with regard to these attributes. Considering knowledge to 'sensitive' information is often important for decisionmaking, counterfactual fairness has been provided as an alternative method to ensure parity treatment. The counterfactual approach builds upon the intuition that a decision is fair towards an individual where the outcome of the decision would be the same in the actual world, as well as the "counterfactual world where the individual would have belonged to a different demographic, sensitive-attribute, group' (Kusner et al. 2017). It has been shown however that such systems can nevertheless lead to discrimination, for instance through the existence of proxies, whereby a sensitive attribute conscious approach could instead allow for the detection of disparity (Ruggieri, Pedreschi, and Turini 2010). ${ }^{7}$ To ensure parity in impact (or impact parity), decision making systems need to avoid disparity in the fraction of users belonging to different sensitive attribute groups (e.g., men, women) that receive beneficial decision outcomes (Zafar, Valera, Rodriguez, and Gummadi 2017). Parity in impact can further be achieved through a variety of methods. First, the machine learning system can aim towards achieving group fairness. Group fairness entails that the prediction of a particular outcome for individuals across groups should have an almost equal probability. In other words, the protected group is statistically treated similar to the general population (Feldman et al. 2014; Dwork et al. 2011, 2017) ${ }^{8}$ Individual fairness aims to treat similar individuals, whereby 'similarity' is defined in relation to the purpose of the task at hand, similarly, i.e. they should receive a similar output by the system.(Dwork et al. 2011). According to Dwork et al. individual fairness can be considered a complement to fair affirmative action, and works as a generalization of the notion of differential privacy (Ibid, 2). Equality of Opportunity techniques, which builds upon individual fairness, ensure that individuals who qualify for a desirable position should have an equal chance of being

${ }^{5}$ Gajane and Pechenizkiy do stress however, that currently no 'consensus' amongst the machine learning community exists yet.

${ }^{6}$ For a more expansive overview of fair machine learning techniques, and the authors cited in the following paragraphs, please also see (Gajane and Pechenizkiy 2017).

${ }^{7}$ Another means to ensure parity treatment is through counterfactual measures (Kusner et al. 2017).

8 According to Dwork et al. group-level definitions of fairness often fail at both fairness and accurate learning: "If two groups actually have different proportions of individuals who are able to pay back their loans, then the accuracy of any learning algorithm will obviously suffer when constrained to predict an equal proportion of paybacks for the two groups. 
correctly classified for this outcome (Hardt, Price, and Srebro 2016; Zafar, Valera, Rodriguez, and Gummadi 2017). One way to formalize equality of opportunity, is through Rawls' notion of 'fair equality of opportunity'. Under the assumption that there is a distribution of natural assets, individuals with a similar level of talent and ability, and a willingness to use them, should have similar prospect of success regardless of their initial place in the social system (Joseph et al. 2016, 3-4). ${ }^{9}$. According to Zafar et al., these methods often present an inherent trade-off to be made between achieving high end accuracy and actually satisfying parity. As accuracy remains crucial for decisionmaking, parity might not be always a goal to strive towards (Zafar, Valera, Rodriguez, Gummadi, et al. 2017).

\subsection{Preference based fairness in Machine Learning}

Considering the inherent trade-off that has to be made between accuracy and parity, Zafar et al. introduced 'preference-based learning' as an alternative (Zafar, Valera, Rodriguez, Gummadi, et al. 2017). The definition of fairness within preference-based approaches has been informed by 'envy-freeness' ${ }^{10}$ literature and game theory (Zafar, Valera, Rodriguez, Gummadi, et al. 2017). Following preference-based machine learning, a fair outcome will be achieved when "given the choice between various sets of decision treatments or outcomes, any group of users would collectively prefer its treatment or outcomes, regardless of the (dis)parity as compared to the other groups (Zafar, Valera, Rodriguez, Gummadi, et al. 2017)." Where preference is defined in terms of treatment, a decision making system should ensure that every sensitive attribute group (e.g., men and women) prefers the set of decisions they receive over the set of decisions they would have received had they collectively presented themselves to the system as members of a different sensitive group. Preferred treatment retains a core fairness property embodied in treatment parity, namely, envy-freeness at the level of user groups. In principle, following this method, no group of (sensitive attribute) users, e.g., men or women, would feel that they would be collectively better off by switching their group membership (in this instance, gender). Preferred impact, on the other hand entails that every sensitive attribute group (e.g., men and women) prefers the set of decisions they receive over the set of decisions they would have received under the criterion of impact parity (Zafar, Valera, Rodriguez, Gummadi, et al. 2017).

\footnotetext{
${ }^{9}$ Jospeh's et al.'s notion of fairness asks that "at every step, a learning algorithm must never "favor" (that is, choose with higher probability) an applicant whose true quality (as determined by their attributes and their group's mapping) is lower than that of another applicant. Our primary interest is in designing learning algorithms that can (provably) converge to optimal decision-making, while being (provably) fair at every step. We seek to quantify the frictions or trade-offs between fairness and fast convergence to optimality, and thus compare (both theoretically and experimentally) our fair learning algorithms with standard algorithms that are unconstrained by fairness."

${ }^{10}$ The envy-test, as introduced by Dworkin, posits that a distribution of goods (or resources) is fair if no other person envies someone else's bundle of resources.
} 


\subsection{Fairness in Machine Learning: Egalitarian?}

Both parity and preference techniques' underpinnings are sufficiently broad in theory. Whereas parity techniques might be most associated with equality as a principle of justice, preference techniques are mainly rooted in envy-free literature and economic game theory. In practice however, both techniques seek to achieve a similar outcome: research focuses primarily on the mitigation of unfair outcomes linked to 'sensitive attributes'. ${ }^{11}$ These attributes mainly correspond to the protected grounds found in nondiscrimination legislation, such as gender and ethnicity. In their own right, the protected grounds correspond to existing societal biases concerning historically disadvantaged groups. Fair machine learning thus mainly targets the known fear that machine learning techniques can reinforce prejudices that persist within society. As a consequence however, and regardless of whether one prefers to implement a parity or preference approach, a strong, albeit narrow and very law-inspired, egalitarian concern is present.

In other words, though reliant on strong normative foundations, current approaches seem to conflate (or equate) fairness with a substantively limited version of 'equality'. ${ }^{12}$ Binns rightfully considers that the methods considered might inadequately consider the wider context in which 'protected' classes became protected and how these classes relate to justice. One of the potential, undesirable consequences, which a sensitive attribute approach can't account for, is the generation of unfair inequalities due to machine learning's inherent differentiating nature. Broader concerns regarding equality as a principle of justice are present due to the capability of machine learning to generate new population segments, composed of an increasing number of attributes and parameters, at a continuous rate (Vedder and Naudts 2017). Yet, these new segments could be composed in seemingly random, yet statistically accurate, ways. The decisions that affect these newly formed groups might still be deemed unfair from an equality perspective. For instance, scholars have argued that new group formations could lead to stereotyping or stigmatization (See inter alia Hildebrandt and Gutwirth 2008; Citron and Pasquale 2014; Zarsky 2014). Furthermore, where these inequalities have an impact on a group-level, they also have an impact on the individual members that constitute the groups (Naudts 2019). ${ }^{13}$

${ }^{11}$ For instance, fairness-aware data mining has been performed on real-world data sets that include 'sensitive' attributes (Friedler et al. 2018). Obviously, the inclusion of such data sets has made these sensitive attributes the main focus of research.

${ }^{12}$ Moreover Hu notes that debates concerning 'AI at-large' is continuously debated, the ethics of individual AIs, such as a machine-learning based classifier, are primarily grounded in utilitarianism: "the right action is the one that maximizes (expected) utility" (Hu Forthcoming). As an example,

${ }^{13}$ Here, one should consider de-invidualization of individuals or their moral equality (Vedder 1999; Delacroix 2018). 


\section{The Articulation of Fairness}

If machine learning is truly to become fair, a broader notion of fairness, or a better understanding of the different existing conceptions of fairness, should be found. As mentioned however, this would first require an articulation of what fairness, in light of automated decision-making and machine learning, entails, having proper consideration for the context and consequences these decisions would have. If one can succeed in these goals, maybe then, the articulation of fairness could serve as a catalyst for new formalization approaches that are better apt at mitigating the potential unfair outcomes of machine learning. ${ }^{14}$

\subsection{Theories of Distributive Justice}

One source where fairness has been articulated extensively is within the field of political philosophy. Political philosophy seeks to determine how the organization of society, which depends upon the interplay and alignment between legal, economic and civil aspects of social life, can be best accustomed to the interests of individuals. According to Pettit, the main goal of political philosophy is to assess rival social arrangements: rival social charters, e.g. theories of justice, are investigated by the political philosopher in an effort to pursue the optimum or to seek justice. ${ }^{15}$ Furthermore, a society's chosen economic, political and social frameworks largely determine the distribution of benefits and burdens of the members within that society. Different structures to these domains will affect people in different ways. Within political philosophy, theories of distributive justice debate the preferred distribution of benefits and burdens across members of society, providing principles, for example equality of opportunity, as moral guidance.

A distinction within distributive justice can be further made between substantive and procedural principles of justice. Whereas procedural justice refers to the procedures that can be used to determine how benefits and burdens of various kinds are allocated to individuals, substantive justice refers to the final allocation, or the outcome of distribution. In simple terms, procedural justice aims to ensure fair treatment, whereas substantive justice aims to ensure a fair share. Distributive theories of justice can then be further distinguished with regard to the weight they attach to either procedures or substantive outcomes. Irrespective of what view one takes, in an ideal scenario of perfect procedural justice, an independent standard would exist for deciding which outcome is just and a procedure that is guaranteed to lead to it. Thus, in a consistent system, a rule

\footnotetext{
${ }^{14}$ It should be noted however that, whereas the articulation of fairness is a precondition for fairness to be formalized, it does not guarantee the latter. Indeed, the aforementioned barriers likely limit the construction of airtight fair systems a priori, due to the often intangible nature of machine learning in combination with its contextual effects over time.

${ }^{15}$ Criteria for justice can be varied, and may include legitimacy (Nozick), welfare (Bentham, Mill) or fairness (Rawls).
} 
that is fair will be set, ultimately leading to an outcome that is fair, in accordance to one specific view of just distribution. ${ }^{16}$

\subsection{Distributive Justice and Machine Learning}

Where machine learning techniques are deployed to guide decision-making in manners that affect the distribution of benefits and burdens across members of society, e.g. with regard to access to resources and opportunities, as is the case in recommender systems, hiring or loan application algorithms, theories of distributive justice in particular might serve as a useful source to begin the articulation of fair machine learning. Applied to algorithmic decision-making, both substantive and procedural distributive justice principles can be reflected upon to evaluate and build fair systems. On the one hand, substantive principles could provide guidance in the assessment related to the outcomes of automated decision-making, the decision as it appears before individuals. ${ }^{17}$ Procedural justice on the other hand might inform questions concerning system functionality, such the logic or general functionality of an automated process, or help inform individual decision-making procedures, such as the rational, reasons and individual circumstances regarding an automated outcome (See also: Wachter, Mittelstadt, and Floridi 2017). Of course, in this case as well, an interplay between both procedures and outcomes exists. For instance, the formalization of fairness, is in principle, a procedural decision-making rule in order to ensure a fair outcome.

Most machine learning research tends to focus on the outcomes. Nevertheless, procedural fairness is starting to be considered by the data science community as a means to structure decision-making systems. Here, procedural fairness, i.e. the fairness of the decision-making process itself, looks at the input features used within decision-making processes and their influence on outcomes, whereby input features are evaluated on the basis of moral judgements made by humans concerning their use (Grgic-Hlaca et al., n.d.). ${ }^{18}$ The formalization of a fairness will nevertheless depend upon the creation of a fair procedural structure or environment capable of achieving fair outcomes. Thus, a convergence between 'substantive', outcome-based fairness and procedural fairness should be sought.

${ }^{16}$ Though procedures in themselves have value, predominantly, procedural justice is considered to be a function of substantive justice, i.e. in function of the outcomes it will produce once applied (Jasso, Törnblom, and Sabbagh 2016; Karen S. Cook and Karen A. Hegtvedt 1983; Lamont and Favor 2017). Nevertheless, it should be noted that an interplay exists between procedural and substantive justice.

${ }^{17}$ Of course, the assessment of when an outcome would be fair, depends upon the perspective decision-makers will take.

${ }^{18}$ Procedural fairness considerations pose the problem that they require an 'understanding of the potential harmful impact on society by unacceptable use of input features in a decision process'. As such, they require background knowledge concerning input features and the societal context. The latter are however not captured in the available data. Therefore, research relies on the moral judgments or instincts of humans to determine when feature use is fair (GrgicHlaca et al., n.d.; as inspired by Yaari and Bar-Hillel 1984). 


\subsection{Beyond Equality}

As indicated, research mainly focuses on achieving fair outcomes with regard to sensitive-attribute groups, i.e. an approach that is both substantive and egalitarian. Even though a strong link between fairness and equality exists within many theories of distributive justice, not all theories of justice promote equality to the same degree. Moreover, if egalitarian principles are equated with fairness, other normative principles at stake, such as autonomy and personal freedom, might become neglected. For instance, in cases where concerns arise regarding nudging, the filter bubble or 'personalized profiling', fairness might be best articulated through liberal or libertarian notions, such as autonomy and self-ownership, or neo-republican theories on non-domination. ${ }^{19}$

Recently, Pettit's neo-republican conception of freedom as non-domination has been considered as a systematic framework that could be used as a means to detect why and how profiling, including profiling based on machine learning, could negatively curtail the freedom of individuals (Gräf 2017). Generally speaking, the enjoyment of freedom requires that no other person or group has the ability to arbitrarily interfere in the affairs of others (Pettite 1996, 1997, 2001, 2012, 2014). As such, freedom requires the 'secure enjoyment of non-domination' (Lovett 2018). Drawing from Pettit's neo-republican conception of freedom as non-domination, Gräf established five criteria to make explicit the problematic nature of certain profiling practices. ${ }^{20}$ Similar discussions could be held concerning the value of Nozick's description of human beings as self-owners (Nozick, State, Anarchy and Utopia). The right to self-ownership "reflects the underlying Kantian principle that individuals are ends and not merely means; they may not be sacrificed or used for the achieving of other ends without their consent. Individuals are inviolable." Where profiling practices are particularly invasive, questions concerning self-ownership could be raised. 'Proprietary' understandings of privacy for instance have found expression in health care and health research contexts (Allen 2016). It remains to be seen however whether these notions can be formalized or not, or whether they can only be protected through organizational or legal measures.

\footnotetext{
${ }^{19}$ Rather than equality, these theories empha-size autonomy, self-ownership and freedom. These concepts, rather than focusing on distributive justice, focus on the individual and are more closely related to the norma-tive underpinnings of privacy and data protection as fundamental rights.

${ }^{20}$ In particular, Gräf distinguishes the following criteria to assess whether or not domination exists a) the presence of intimidation and invigilation when profiling enables or enhances capacities to monitor persons, b) via automated manipulation of a person's choices, c) via active interference with the scope of action of persons who are identified with the help of automated profiling, d) via automated adjustments to a person's informational or material environment, and in cases of the personalities of a person's environment, when the person has no control over the way this personalization happens.
} 


\subsection{Barriers towards Fair Machine Learning: The Contextual Challenges of Technology and Automation}

The articulation of fair machine learning does not simply depend upon the investigation of analytical, philosophical constructs. ${ }^{21}$ Discussions concerning fairness should not be held in a vacuum: it is important to consider the particular reality within which algorithms and machine learning techniques operate. Both the context in which algorithms are deployed, as well as the contexts and consequences they generate through their functioning, should be taken into the discussion. As Verbeek indicates: technology works as an active mediator, they co-shape people's being in the world. Technology alters the perception and actions, experience and existence of those who interact with it (Verbeek, 2006). Not only does technology alter the perception of those it interacts with, it might also alter societal structures. Algorithms take meaning from the outside world, but they also shape meaning (Roberge and Melançon 2017, 3). For instance, machine learning might uncover and re-shape current societal strata (Vedder and Naudts 2017, Naudts 18). ${ }^{22}$

Related to the latter, it is also important to consider the effects that machine learning processes may have over time. For instance, new stereotypes, not linked to sensitiveattribute groups, but that result out of inequalities generated by machine learning, are one instance where fairness should be considered in a broader framework, taking into account contextual changes over time. Liu et al. point out that current machine learning research mainly focus on static objectives "defined on a snapshot of the population at one instant in time", which inadequately takes into account changes deployed automated processes might have on the underlying populations over time (Liu et al. 2018). Many decisions aided through machine learning however are consequential in nature: lending practices can shift the distribution of debt and wealth in the population, whereas recruiting tools allocate opportunities (Ibid, 1). In this regard, Hu considers that current research has implicitly assumed that 'fair algorithms can operate self-sufficiently without reference to humans or context', which, in turn, might limit a holistic approach of fair machine learning. In sum: "those interested in transformative impact in the area of fair and accountable machine learning must move towards studying these processes in vivo, in the messy, socio-technical contexts in which they inevitably exist (Veale, Van Kleek, and Binns 2018).”

${ }^{21}$ Binns rightfully notes that where abstract principles of political philosophy are applied in an effort to analyze particular instances of unfair inequalities, theoretical concepts need to be supplemented with empirical claims concerning the 'how and why' these inequalities came to be (Binns 2018, 8). The latter however is not the domain of political philosophy. Rather, sociology, history and economics might increase our understanding on the specific circumstances under which groups become unfairly disadvantaged (Hooks 1992, Fang and Moro 2010, Binns 2018)

${ }^{22}$ Furthermore, where fairness in decision-making is concerned, the technological context poses an additional challenge concerning questions of accountability and responsibility (For an overview, see inter alia Noorman 2018 or Nissenbaum). 
Moral judgments concerning fairness might also depend on the overall context in which machine learning processes are deployed. Loan application algorithms might be subject to higher scrutiny because the consequences they might have on an individual's life path are bigger. Here fairness criteria that result in higher protection for the individual could be preferred. Whereas the same criteria could deem a shopping platform's recommender system as unfair for individuals involved, precedence might need to be given to the commercial interests of the platform. Similarly, one might favor the use of predictive learning systems in the context of national security, but not for predictive policing in general.

Beyond the contextual elements that are shaped and generated by machine learning, one should also consider the particular area that is the subject of ethical investigation. For instance, Hu notes that, while the ethics of 'AI at-large', including machine learning in general, are continuously debated, discussions concerning the ethics of 'individual AIs', such as a single machine-learning based classifier, are less prevalent. Focus on one area could nonetheless influence the overall normative foundations. Hu considers that the approach taken with regard to individual AIs, is a utilitarian one: "the right action is the one that maximizes (expected) utility" (Hu Forthcoming). Such assumptions however bear strong consequences for the overall outcome of artificial intelligence. Here, the danger exists that, in favor of utility, the broader context of machine learning becomes underrepresented, and that the fairness one sought to achieve is further pushed into one direction. Likewise, Dwork and Ilvento note that research too often considers cases of single classifiers in isolation. Fairness however is not a property to be considered in isolation, but with regard to systems as a whole, in interaction with other systems (Dwork and Ilvento, 2018).

\section{The Articulation and Formalization of Fairness: Facilitating Accountability?}

Despite the difficulties that exist in articulating and formalizing fairness, the articulation, and subsequent formalization of fairness, can nonetheless become a crucial step towards the creation of more accountable systems, and the accountability of actors who use machine learning for decision-making purposes. ${ }^{23}$ Accountability, in general terms, and for the purposes of this paper, can be described as the ability of decision-makers to provide good reasons and justifications with regard to the decisions they take to a (hypothetical forum) of persons (Vedder and Naudts 2017). As such, accountability has two main components: justifiability and answerability (Leonelli 2016). Accountability thus requires decision-makers to reflect upon the reasons why a decision is to be made, to consider the alternatives and to justify why a given decision was made in preference

\footnotetext{
${ }^{23}$ For instance, the FATML community lists fairness, amongst responsibility, explainability, accuracy and auditability as one of the key guiding principles for accountable systems ( FAT ML' n.d.).
} 
over the existing alternatives. Moreover, the explanation for doing so must be externalized towards an external forum, such as a public authority, ethics board or society at large. Where decision-makers rely on automated processes, such as machine learning, the claims, actions and decisions made on the basis of these processes should be subject to a similar review.

Concerning the justifiability component of accountability, if processes can be designed to be both procedurally and substantively fair, decision-makers should be encouraged to consider the different normative frameworks available to them. As different conceptions of fairness exist, decision-makers would then have to make a decision as to which conception of fairness they wish to integrate. At the same time, decisionmakers who decide to deploy machine learning processes, should have awareness concerning the notions of fairness that were integrated. Of course, fair machine learning does not imply that decision-makers should only make decisions in the benefit of society at large. Yet, where automated processes are deployed to achieve a particular goal, for instance to protect national security or to secure commercial viability, decisionmakers should still ensure that they integrate systems that can achieve those goals in a fair manner. Having integrated fairness, either through its articulation or formalization, the underlying normative framework can also facilitate decision-makers in externalizing the underlying reasoning behind their decisions. First, the notion of fairness used can be made public. It would be particularly useful if those affected by a decision could be provided with an explanation with regard to the conceptions of fairness that were integrated into the decision, and how the decision was informed by the chosen notion of fairness. This might also allow individuals to understand the overall decision better. For instance, they might indicate why a certain parameter was relevant for a given decision, and why it was fair to achieve the goal of the decision-maker. In this regard, the articulation and formalization of fairness could enable an ex-post review on a technical, ethical and legal level, by providing a framework upon which practices can be judged by. Where the final outcome of a machine learning process does not longer correspond to the principles one sought to achieve, i.e. the outcome does not correspond to what the programmers deemed fair, or where embedded principles were, at a later stage, deemed inappropriate by civil society, e.g. society at large favours a different conception of fairness for the decision-making process, it could facilitate the revision (or penalization) of the automated system. ${ }^{24}$

Given the many actors involved in developing technological processes, it remains difficult to allocate accountability within the technological environment (See inter alia Nissenbaum, n.d.; Leonelli 2016; Johnson and Powers 2005; Verbeek 2006; Floridi 2013). ${ }^{25}$ Data Protection laws however, and the GDPR in particular have ascribed the

\footnotetext{
${ }^{24}$ Here, the articulation of fairness, as a separate process next to the formalization thereof, becomes increasingly important. Even where fairness cannot be programmed, having articulated fairness in light of automated decision-making processes, still allows the evaluation of automated processes.

${ }^{25}$ Similar 'allocation' problems exist with regard to moral responsibility, associated with accountability, within a technological environment (Noorman 2018).
} 
responsibility for accountability onto the data controller, i.e. the entity who determines the means, e.g. machine learning, and purposes, e.g. the decision to be made, for data processing. Furthermore, if fair machine learning can be considered an important step towards accountability, the GDPR might become a catalyst for the integration of a legal obligation to implement fair machine learning. Here, it is important to note that accountability, as an overarching principle, is embedded throughout the GDPR, for instance through mechanisms like data protection by design, transparency and information requirements the Data Protection Impact Assessment. The Data Protection Impact Assessment for instance, which will likely have to be performed where machine learning technologies are deployed for automated decision-making purposes, not only requires data-controllers to identify the risks to the fundamental rights and interests of natural persons, but also to implement effective countermeasures to address these risks. In this regard, fair machine learning could be considered an effective means to limit the potential negative effects of automated decision-making on fundamental rights and interests.

Even though the data controller can be considered accountable, he should not be solely responsible for ensuring fair machine learning. According to Nissenbaum, accountability applies to all stakeholders involved in the decision-making process. Accountability thus requires an organizational context, one in which answerability works to entice people to pay greater attention to system safety, reliability and sound design, in order to establish a culture of accountability (Nissenbaum, n.d.; Noorman 2018). As an example, where decision-makers rely on third-party systems, there's a responsibility for the decision-maker to rely on soundly designed learning systems. In their own regard, designers should ensure that their algorithms are tested for the presence of potential biases, and trained via accurate, non-biased data. Whereas the articulation of fairness might remain the area of ethical and legal academics, data scientists could further strengthen the design of their processes to be more holistic towards fairness. In addition, society at large, or individuals affected, could contribute to more accountable systems.

\subsection{Citizen Participation for the Creation Accountable Systems}

Providing insight, through transparency and accountability mechanisms, into the way machine learning processes operate, does not necessarily increase the responsibility of data subjects vis-à-vis the processes to which they will be made subject to. From an accountability perspective, it is nevertheless important that individuals are informed about the elements that constitute decision-making processes: they are part of the forum. Furthermore, it provides the possibility for individuals affected an opportunity to be more involved in the decision-making processes. In this regard, it is important to note some recent developments regarding the value of justice, as it is perceived by data 
subjects. Research shows that individuals do consider the justice-related aspects of algorithmic decision-making processes (Binns et al. 2018). ${ }^{26}$ An analysis of citizens' perceptions on fairness might also help guide the creation of fair systems. A recent study indicates that individuals are informed by a variety of feature properties, including relevancy, privacy and reliability, in their moral judgements concerning the desirability of using certain features in automated decision-making (Grgic-Hlaca et al. 2018b) ${ }^{27}$ In other words, the perceived fairness and moral judgments of individuals can be used as a means to evaluate and create fair systems. ${ }^{28}$ Whereas the responsibility of creating fair systems depends upon decision-makers, individuals could thus shape the definition of these systems. Nevertheless, it does bear mentioning however that perceived fairness does not always equate fair systems. Moreover, research also notes that on the individual level, people's fairness judgments diverge considerably (Grgic-Hlaca et al. 2018b).

\section{$5 \quad$ Concluding Remarks}

The articulation, and subsequent formalization of fairness (where possible), is but one part of the puzzle. First, the contextual barriers that surround machine learning must be overcome in order to have a better understanding of the changes machine learning processes might bring about over time. In turn, discussions concerning the latter might inform future developments regarding the articulation of fairness within the context of machine learning, the ethical domain, and the formalization thereof, the technical domain. In any case, a multi-stakeholder approach will be needed in order to better gauge these contextual changes and how they might alter our perceptions of what is fair and what not. Second, even though fairness could inform the creation of accountable systems, and could make decision-makers more accountable, fairness in itself does not equate accountability. For instance, fair systems might not always be interpretable or 'explainable'. Nevertheless, an increased level of awareness, or emphasis on fairness, could stimulate decision-makers to better consider the societal consequences of the means they choose to achieve their goals. However, in order to create an organizational context, capable of cultivating a culture of accountability, the importance of fairness within machine learning should be further encouraged, also on a policy level.

${ }^{26}$ Nevertheless, when it comes to the provision of an explanation provided to individuals, the capability to evaluate the fair character of decision-making systems depends upon the context in which machine learning are deployed (Binns et al. 2018).

${ }^{27}$ Research seems to that individuals perceive fairness on a multi-dimensional level, beyond discrimination.

${ }^{28}$ For instance, feature volitionality looks whether an input feature, relevant to a decision, is the result of volitional decisions made by the individual, or of circumstances beyond their control (Grgic-Hlaca et al., n.d., 2018b). Volitionality as a parameter to measure fairness is interesting as it includes links to political philosophy, and luck equality in particular, where 'volition' or choice is considered an important element when determining whether inequalities are unfair and should thus be compensated. 


\section{Acknowledgments}

This paper was supported by the VICTORIA project which has received funding from the European Union's Horizon 2020 research and innovation programme under grant agreement No 740754, and by imec, Flanders' digital research centre and incubator.

\section{$7 \quad$ References}

Binns, Reuben. 2018. 'Fairness in Machine Learning: Lessons from Political Philosophy'. In Proceedings of Machine Learning Research. New York City.

Binns, Reuben, Max Van Kleek, Michael Veale, Ulrik Lyngs, Jun Zhao, and Nigel Shadbolt. 2018. "IIt's Reducing a Human Being to a Percentage"; Perceptions of Justice in Algorithmic Decisions'. ArXiv:1801.10408 [Cs], January. https://doi.org/10.1145/3173574.3173951.

Citron, Daniel Keats, and Frank Pasquale. 2014. 'The Scored Society: Due Process for Automated Predictions'. Wash. L. Rev. 89 (1): 1-33.

Colonna, Liane. 2013. 'A Taxonomy and Classification of Data Mining'. Science and Technology Law Review 16: 63.

Delacroix, Sylvie. 2018. 'Pervasive Data Profiling, Moral Equality and Civic Responsibility.' In . London.

Diakopoulos, Nicholas. 2015. 'Algorithmic Accountability: Journalistic Investigation of Computational Power Structures'. Digital Journalism 3 (3): 398-415. https://doi.org/10.1080/21670811.2014.976411.

Dwork, Cynthia, Moritz Hardt, Toniann Pitassi, Omer Reingold, and Rich Zemel. 2011. 'Fairness Through Awareness'. ArXiv:1104.3913 [Cs], April. http://arxiv.org/abs/1104.3913.

Dwork, Cynthia, and Christina Ilvento. n.d. 'Group Fairness Under Composition', 6. . n.d. 'Individual Fairness Under Composition', 6.

Dwork, Cynthia, Nicole Immorlica, Adam Tauman Kalai, and Max Leiserson. 2017. 'Decoupled Classifiers for Fair and Efficient Machine Learning'. ArXiv:1707.06613 [Cs], July. http://arxiv.org/abs/1707.06613.

Feldman, Michael, Sorelle Friedler, John Moeller, Carlos Scheidegger, and Suresh Venkatasubramanian. 2014. 'Certifying and Removing Disparate Impact'. ArXiv:1412.3756 [Cs, Stat], December. http://arxiv.org/abs/1412.3756.

Floridi, Luciano. 2013. 'Distributed Morality in an Information Society'. Science and Engineering Ethics 19 (3): 727-43. https://doi.org/10.1007/s11948-012-9413-4.

Friedler, Sorelle A., Carlos Scheidegger, Suresh Venkatasubramanian, Sonam Choudhary, Evan P. Hamilton, and Derek Roth. 2018. 'A Comparative Study of Fairness-Enhancing Interventions in Machine Learning'. ArXiv:1802.04422 [Cs, Stat], February. http://arxiv.org/abs/1802.04422.

Gajane, Pratik, and Mykola Pechenizkiy. 2017. 'On Formalizing Fairness in Prediction with Machine Learning'. ArXiv:1710.03184 [Cs, Stat], October. http://arxiv.org/abs/1710.03184.

Grgic-Hlaca, Nina, Elissa M. Redmiles, Krishna P. Gummadi, and Adrian Weller. 2018a. 'Human Perceptions of Fairness in Algorithmic Decision Making: A Case Study of 
Criminal Risk Prediction'. In , 903-12. ACM Press. https://doi.org/10.1145/3178876.3186138.

. 2018b. 'Human Perceptions of Fairness in Algorithmic Decision Making: A Case Study of Criminal Risk Prediction'. In , 903-12. ACM Press. https://doi.org/10.1145/3178876.3186138.

Grgic-Hlaca, Nina, Muhammad Bilal Zafar, Krishna P Gummadi, and Adrian Weller. n.d. 'Beyond Distributive Fairness in Algorithmic Decision Making: Feature Selection for Procedurally Fair Learning', 10.

Hardt, Moritz, Eric Price, and Nathan Srebro. 2016. 'Equality of Opportunity in Supervised Learning'. ArXiv:1610.02413 [Cs], October. http://arxiv.org/abs/1610.02413.

Hildebrandt, Mireille, and Serge Gutwirth, eds. 2008. Profiling the European Citizen: Cross-Disciplinary Perspectives. New York: Springer.

$\mathrm{Hu}$, Lily. Forthcoming. Justice Beyond Utility in Artificial Intelligence. New Orleans: AAAI/ACM.

Jasso, Guillermina, Kjell Y. Törnblom, and Clara Sabbagh. 2016. 'Distributive Justice'. In Handbook of Social Justice Theory and Research, edited by Clara Sabbagh and Manfred Schmitt, 201-18. New York, NY: Springer New York. https://doi.org/10.1007/978-1-4939-3216-0_11.

Johnson, Deborah G., and Thomas M. Powers. 2005. 'Computer Systems and Responsibility: A Normative Look at Technological Complexity'. Ethics and Information Technology 7 (2): 99-107. https://doi.org/10.1007/s10676-005-4585-0.

Joseph, Matthew, Michael Kearns, Jamie Morgenstern, Seth Neel, and Aaron Roth. 2016. 'Fair Algorithms for Infinite and Contextual Bandits'. ArXiv:1610.09559 [Cs], October. http://arxiv.org/abs/1610.09559.

Karen S. Cook, and Karen A. Hegtvedt. 1983. 'Distributive Justice, Equity, and Equality'. Annual Review of Sociology, 217-41.

Kusner, Matt J., Joshua R. Loftus, Chris Russell, and Ricardo Silva. 2017. 'Counterfactual Fairness'. ArXiv:1703.06856 [Cs, Stat], March. http://arxiv.org/abs/1703.06856.

Lamont, Julian, and Christi Favor. 2017. 'Distributive Justice'. In The Stanford Encyclopedia of Philosophy, edited by Edward N. Zalta, Winter 2017. Metaphysics Research Lab, Stanford University. https://plato.stanford.edu/archives/win2017/entries/justicedistributive/.

Leonelli, Sabina. 2016. 'Locating Ethics in Data Science: Responsibility and Accountability in Global and Distributed Knowledge Production Systems'. Philosophical Transactions of the Royal Society A: Mathematical, Physical and Engineering Sciences 374 (2083): 20160122. https://doi.org/10.1098/rsta.2016.0122.

Liu, Lydia T., Sarah Dean, Esther Rolf, Max Simchowitz, and Moritz Hardt. 2018. 'Delayed Impact of Fair Machine Learning'. ArXiv:1803.04383 [Cs, Stat], March. http://arxiv.org/abs/1803.04383.

Naudts, Laurens. 2019. 'How Machine Learning Generates Unfair Inequalities and How Data Protection Instruments May Help in Mitigating Them.' In The Internet of Bodies, 14. Hart Publishing.

Nissenbaum, Helen. n.d. 'Computing and Accountability', 9.

Noorman, Merel. 2018. 'Computing and Moral Responsibility'. In The Stanford Encyclopedia of Philosophy, edited by Edward N. Zalta, Spring 2018. Metaphysics Research Lab, Stanford University. https://plato.stanford.edu/archives/spr2018/entries/computing-responsibility/. 
'Principles for Accountable Algorithms and a Social Impact Statement for Algorithms :: FAT ML'. n.d. Accessed 4 August 2018. https://www.fatml.org/resources/principlesfor-accountable-algorithms.

Roberge, Jonathan, and Louis Melançon. 2017. 'Being the King Kong of Algorithmic Culture Is a Tough Job after All: Google's Regimes of Justification and the Meanings of Glass'. Convergence: The International Journal of Research into New Media Technologies 23 (3): 306-24. https://doi.org/10.1177/1354856515592506.

Robinson, David, Harlan Yu, and Aaron Rleke. 2014. 'Civil Rights, Big Data, and Our Algorithmic Future'. http://centerformediajustice.org/wp-content/uploads/2014/10/Civil-Rights_Big-Data_Our-Future.pdf.

Ruggieri, Salvatore, Dino Pedreschi, and Franco Turini. 2010. 'Data Mining for Discrimination Discovery'. ACM Transactions on Knowledge Discovery from Data 4 (2): 140. https://doi.org/10.1145/1754428.1754432.

Veale, Michael, Max Van Kleek, and Reuben Binns. 2018. 'Fairness and Accountability Design Needs for Algorithmic Support in High-Stakes Public Sector Decision-Making'. In , 1-14. Montréal: ACM Press. https://doi.org/10.1145/3173574.3174014.

Vedder, Anton. 1999. 'KDD: The Challenge to Individualism'. Ethics and Information Technology 1 (4): 275-281.

Vedder, Anton, and Laurens Naudts. 2017. 'Accountability for the Use of Algorithms in a Big Data Environment'. International Review of Law, Computers \& Technology 31 (2): 206-24. https://doi.org/10.1080/13600869.2017.1298547.

Verbeek, Peter-Paul. 2006. 'Materializing Morality: Design Ethics and Technological Mediation'. Science, Technology, \& Human Values 31 (3): 361-80.

https://doi.org/10.1177/0162243905285847.

Wachter, Sandra, Brent Mittelstadt, and Luciano Floridi. 2017. 'Why a Right to Explanation of Automated Decision-Making Does Not Exist in the General Data Protection Regulation'. International Data Privacy Law 7 (2): 76-99.

Zafar, Muhammad Bilal, Isabel Valera, Manuel Gomez Rodriguez, and Krishna P. Gummadi. 2017. 'Fairness Beyond Disparate Treatment \& Disparate Impact: Learning Classification without Disparate Mistreatment'. ArXiv:1610.08452 [Cs, Stat], 1171-80. https://doi.org/10.1145/3038912.3052660.

Zafar, Muhammad Bilal, Isabel Valera, Manuel Gomez Rodriguez, Krishna P. Gummadi, and Adrian Weller. 2017. 'From Parity to Preference-Based Notions of Fairness in Classification'. ArXiv:1707.00010 [Cs, Stat], June. http://arxiv.org/abs/1707.00010.

Zarsky, Tal Z. 2014. 'Understanding Discrimination in the Scored Society'. Wash. L. Rev. 89 (4): 1375-1412. 